\title{
GLAD!
}

Revue sur le langage, le genre, les sexualités

$06 \mid 2019$

Varia

\section{Agentivité sexuelle des femmes dans les films pornographiques critiques réalisés par des femmes}

Women's Sexual Agency in Critical Pornographic Films Made by Women

Julie Lavigne, Myriam Le Blanc Elie et Sabrina Maiorano

\section{OpenEdition}

Édition électronique

URL : http://journals.openedition.org/glad/1476

DOI : $10.4000 /$ glad. 1476

ISSN : 2551-0819

Éditeur

Association GSL

Référence électronique

Julie Lavigne, Myriam Le Blanc Elie et Sabrina Maiorano, «Agentivité sexuelle des femmes dans les films pornographiques critiques réalisés par des femmes », GLAD! [En ligne], 06 | 2019, mis en ligne le 01 juillet 2019, consulté le 17 décembre 2020. URL : http://journals.openedition.org/glad/1476 ; DOI https://doi.org/10.4000/glad.1476

Ce document a été généré automatiquement le 17 décembre 2020.

La revue GLAD! est mise à disposition selon les termes de la Licence Creative Commons Attribution Pas d'Utilisation Commerciale - Pas de Modification 4.0 International. 


\title{
Agentivité sexuelle des femmes dans les films pornographiques critiques réalisés par des femmes
}

\author{
Women's Sexual Agency in Critical Pornographic Films Made by Women
}

Julie Lavigne, Myriam Le Blanc Elie et Sabrina Maiorano

That's what really bothers you isn't it? The one-night stand? Man fucks woman. Subject man, verb fucks, object woman. That's okay. Woman fucks man. Woman subject, man object. That's not so comfortable for you is it?

Réplique du personnage de Stella Gibson dans la série télévisuelle The Fall (2013-2016).

1 Il est certes surprenant, mais pas anodin, de retrouver une telle réplique dans une série policière anglaise de type quality TV (McCabe et Akass 2007). Cette réplique, qui paraphrase le «Man fucks woman; subject verb object» de MacKinnon (1982: 541), aborde de front le script hétérosexuel traditionnel ${ }^{1}$ - où l'homme est le sujet agissant et la femme, l'objet du désir - décrié par les féministes depuis Simone de Beauvoir (1949). Or, si une telle réplique se retrouve au sein d'un objet culturel commercial, c'est symptomatique que de nouveaux scripts sexuels émergent où les femmes agissent sexuellement (ou font preuve d'agentivité sexuelle). Et, comme en témoigne cette citation, l'émergence de nouveaux scripts sexuels, qui cohabitent avec les scripts hétéro traditionnels, ne se fait pas sans heurts. De même, retrouver de tels propos dans une série aussi populaire que The Fall contribue à visibiliser ces nouveaux scripts sexuels, à diversifier le répertoire des scénarios existants et à permettre de transformer les conditions mêmes du vécu sexuel. Cette affirmation s'avère d'autant plus pertinente quand les scripts sexuels émergents se trouvent dans un médium qui diffuse des scripts aussi explicites que la pornographie.

2 Dans cet article, il sera question de faire état des résultats du projet Pornographie critique, féministe, queer et post-pornographie: contours d'une pratique émergente (CRSH, 
2013-2016), qui vise à analyser un courant de la pornographie qui affirme produire une pornographie différente et critique de la pornographie commerciale faite par et pour des hommes. Nous avons ainsi analysé la production de 38 films réalisés par des femmes cisgenres et personnes transgenres pour un public de femmes cis ou de personnes trans lesbiennes, hétérosexuelles ou queer ${ }^{2}$. Plus spécifiquement, nous avons examiné les scripts sexuels et de genre mis en scène, et les discours sur les sexualités des femmes qui sont véhiculés par ces productions: notamment sur l'agentivité sexuelle et la mise en scène du désir, du plaisir et de l'orgasme. L'article aborde la question précise, mais complexe, de l'agentivité sexuelle. Dans un premier temps, les différentes définitions théoriques de l'agentivité sexuelle seront recensées et les limites $\mathrm{du}$ concept seront exposées. Cette première partie dépasse un simple état des connaissances sur le concept pour constituer une réflexion critique plus élaborée. Dans un second temps, les marqueurs d'agentivité identifiés dans les films du corpus seront expliqués. Il sera notamment question des patrons de consentement, de la communication des préférences sexuelles et des transformations du script hétérosexuel traditionnel, condition de possibilité de l'agentivité sexuelle des femmes. Dans le contexte actuel de (re)politisation des enjeux liés à la sexualité, avec la vague de dénonciations des violences sexuelles du mouvement \#moiaussi ${ }^{3}$, la mise en exergue par de nombreuses féministes de l'omniprésence de la culture du viol et une préoccupation grandissante pour la question du consentement sexuel, il appert essentiel de revenir sur la notion d'agentivité sexuelle.

\section{Pourquoi étudier la pornographie?}

Avant d'aborder la question de l'agentivité sexuelle, il importe de préciser à quel titre la pornographie est étudiée dans le contexte de cet article. Depuis les travaux de Linda Williams (1989) suivant la théorisation de la sexualité de Foucault (1976), les pornographies hardcore sont interprétées comme un dispositif de la scientia sexualis ${ }^{4}$ (Foucault 1976; Williams 1989; Lavigne 2009; Lavigne 2014). Selon Williams, il est possible de définir de manière minimale et la plus neutre possible la pornographie hardcore comme suit: "une représentation visuelle (et parfois auditive) de corps vivants et en mouvement performant des actes sexuels explicites et usuellement non simulés, ayant comme but premier d'exciter le spectateur." (Williams 1989: 29-30, notre traduction). En effet, pour Williams, la pornographie hardcore a donc pour but d'exciter le spectateur, mais prétend aussi exposer du "vrai sexe», des actes sexuels non simulés, principalement par le biais des gros plans de pénétrations et de la visibilité de l'éjaculation.

Bien que cet article ne s'inscrive pas dans une étude de la réception comme telle, il est difficile dans une perspective constructiviste de la sexualité de nier l'influence des divers discours médiatiques et pornographiques sur le vécu sexuel (de Lauretis 1987; Foucault 1976; Gagnon, 2008; Gagnon et Simon 1973 ; Rubin 1984; Scott et al. 2010). Dans cette optique, il s'avère prometteur d'étudier la sexualisation de l'espace public à partir de la perspective de Simon et Gagnon (Gagnon 2008 ; Gagnon et Simon 1973) qui considèrent que la sexualité se vit majoritairement à travers les scripts sexuels préexistants, bien que ceux-ci puissent être l'objet de réécritures. Dans cette approche, la sexualité en soi n'existe pas, elle s'acquiert entre autres par le biais des scénarios culturels et la pornographie a été le "premier exemple de scénario culturel [...] 
définissant comment les gens doivent et ne doivent pas se conduire sexuellement " (Gagnon 2008: 82). La pornographie possède cependant une influence indirecte seulement, car les scénarios culturels doivent toujours transiter par la vie intrapsychique d'un individu qui fait consciemment ou inconsciemment le tri parmi des scénarios concurrents qui lui sont exposés par différents médias, la famille, les pairs, l'école, etc., ainsi que par ses propres expériences. L'objectif n'est pas de mesurer l'impact des scripts sexuels genrés diffusés par les pornographies, car la recherche en communication a changé de paradigme, délaissant l'identification de potentiels effets directs pour passer à un " paradigme des effets limités » (Proulx et Bélanger 2003), où le public est considéré comme actif et critique (Hall 1980 ; Radway 1984). L'influence des scénarios culturels proposés ne relève pas d'un simple apprentissage social direct, mais il s'agit plutôt d'un bassin de scripts qui alimentent les fantasmes (scripts intrapsychiques) et permettent d'élargir ou de limiter le répertoire de gestes, de combinaisons de gestes et de réactions dans les rencontres. La théorie des scripts établit ainsi des conditions de possibilités davantage que des prescriptions strictes, permettant de dépasser la thèse d'un effet direct des images sur les comportements telle qu'argumentée par le féminisme antipornographie renouvelé5 (Bridges 2016; Smith et Attwood 2012 ; Dines 2010).

5 Les pornographies participent ainsi, avec ces divers scénarios, à l'établissement des conditions de possibilités contemporaines du vécu sexuel; elles s'insèrent dans ce système qui régule la vie sexuelle, dans le dispositif de la sexualité. Et comme nous postulons, à l'instar de Judith Butler (1990a/2006 : 106), l'impossibilité d'une sexualité pré ou hors du système patriarcal et du dispositif de la sexualité, il appert tout à fait logique pour des féministes ou des personnes s'affichant comme queer d'investir le milieu de la pornographie pour en proposer une version plus en adéquation avec leurs vécus sexuels et leurs valeurs. Ainsi, pour avoir une meilleure compréhension de la diversité sexuelle des femmes et des discours normatifs sur leur sexualité, il devient primordial d'analyser comment les films pornographiques critiques jouent avec la norme pornographique (telle que décrite par Williams 1989) afin de proposer une pensée critique sur une diversité de pratiques sexuelles pour les femmes cis et les personnes trans.

6 L'intérêt d'analyser cette production vient du fait qu'il s'agit d'un ensemble de pornographies qui répond à la fois aux scripts hétérosexuels traditionnels véhiculés dans les médias généralistes (Kim et al. 2007; Kirsch et Murnen 2015 ; Seabrook et al. 2017), et aux pornographies mainstream où les femmes sont certes agissantes, mais où leur plaisir est secondaire par rapport au plaisir de l'homme, ou présenté de manière partielle, stéréotypée et à partir d'un point de vue masculin. Certes, les pornographies se sont diversifiées depuis les années 1980, posant notamment la question des conditions de travail éthiques, ou mettant en scène une diversité corporelle accrue, au point de rendre la distinction entre pornographie mainstream et pornographie féministe ou alternative très poreuse (voir entre autres Tibbals 2014 et Maina 2014). Cependant, il s'agit encore d'un produit conçu par et pour des hommes, qui s'avère souvent incapable de témoigner adéquatement de l'éventail de la sexualité des femmes cis ou des personnes trans ${ }^{6}$ comme en témoignent beaucoup de productrices et réalisatrices de films pornographiques féministes (Le Blanc, Lavigne et Maiorano 2017 ; Lavigne 2007). La pornographie féministe se donne comme mandat de placer le plus souvent les femmes cis ou les personnes trans, qu'elles soient lesbiennes, bisexuelles, fluides, pansexuelles ou queer, comme des personnages principaux (Le Blanc, Lavigne et 
Maiorano 2017). Notre hypothèse de recherche est qu'en raison de cette différence, la pornographie féministe serait un lieu de transformation des scripts hétérosexuels traditionnels tels qu'ils sont diffusés dans les médias, d'une présence accrue de représentations agentives de la sexualité des femmes et d'une représentation plus « authentique » et plus diversifiée du plaisir et de la jouissance de celles-ci.

7 Cette hypothèse a d'ailleurs été, différemment certes, traitée par Corsianos (2007), Attwood (2011) et Liberman (2015). Corsianos propose de se demander si l'agentivité sexuelle est possible chez les femmes, car elles sont inondées de représentations patriarcales de la sexualité tout particulièrement issues de la pornographie mainstream. Sans avoir démontré empiriquement le phénomène, elle postule que la pornographie est un frein clair au développement de l'agentivité des femmes qui n'arrivent plus à résister au discours hétérosexiste et patriarcal de la pornographie. Pour l'auteure, la création de pornographie alternative et de scénarios culturels différents s'avère être la voie à suivre pour changer structurellement le vécu sexuel des femmes. Pour sa part, Attwood (2011) soutient que les nouvelles pratiques de webcam et les pornographies alternatives comme SuicideGirls ou Furry $\mathrm{Girl}^{7}$ ont transformé les modes de représentation des corps féminins traditionnels suscitant l'objectivation sexuelle, pour devenir un mode de présentation du soi sexuel performatif. En utilisant des outils technologiques, artistiques et de communication pour offrir une présentation de soi qui s'inscrit dans leurs vies, leurs relations et leurs idées politiques, ces productrices d'images pornographiques font preuve d'une réelle agentivité sexuelle (Attwood 2011 : 212). Liberman (2015), pour sa part, a interrogé des productrices, des actrices et des consommatrices de pornographie féministe. Ses résultats montrent que la pornographie féministe devient un espace hétérotopique où cohabitent différentes positions sur la sexualité féminine, parfois oppositionnelles: par exemple, les productions de films peuvent traiter le corps comme une marchandise ou justement effectuer des choix de production pour le bien-être des actrices (amélioration des conditions de travail, sexe sécuritaire) (Liberman 2015). Pour Liberman : « ... feminist pornography offers the chance to engage directly with agentic explicit representations of female desire from third-wave, sex-positive feminist perspective " (Liberman 2015 : 188). Bien qu'elles n'aient pas analysé directement le contenu visuel et sexuel des films pornographiques, celles qui produisent, performent et consomment cette pornographie la considèrent comme proposant des modèles de femmes sexuellement agentives. Ces résultats encouragent ainsi une analyse détaillée des films ciblés entre autres par les recherches de Liberman.

\section{Agentivité sexuelle : tour d'horizon du concept}

8 Nous aimerions dans un premier temps faire le point sur les différentes définitions de l'agentivité sexuelle. Succinctement, le concept sert à décrire le pouvoir d'agir des femmes et leur possibilité d'adopter une posture de sujet lors d'interactions à caractère sexuel (Attwood 2007; Gill 2008; LeMoncheck 1997). Il s'agit d'un concept qui a été maintes fois travaillé dans un contexte anglo-saxon, mais peu abordé en français. Marie-Ève Lang (2011) sera la première à rédiger en français une revue de littérature exhaustive sur le sujet. Comme en témoigne son article, il s'agit d'un concept polysémique qui est loin de faire consensus. Bien qu'elle fasse le tour des différentes définitions, Lang ne propose pas une définition réellement opérationnelle de 
l'agentivité sexuelle. Dans son article, on peut repérer au moins sept éléments de définition provenant de deux études différentes : l'idée de posséder son corps et sa sexualité ; la prise d'initiative ; la conscience du désir ; le sentiment de confiance et de liberté ; le contrôle du désir et du plaisir ; avoir droit au désir et au plaisir.

Outre la polysémie du concept, il importe de spécifier que l'agentivité sexuelle est presque toujours réfléchie dans le contexte des relations hétérosexuelles de jeunes femmes ou adolescentes cisgenres blanches. La définition du concept dans sa délimitation actuelle est hétéronormative (l'agentivité sexuelle n'a pas lieu d'être analysée si elle n'est pas en opposition avec l'assertivité d'un partenaire masculin); âgiste (les jeunes femmes sont jugées peu agentives contrairement aux femmes plus matures, et les femmes âgées sont invisibilisées) ; cisnormative (aucune étude ne porte sur l'agentivité sexuelle des personnes trans); vanille (notamment dans les relations BDSM, qualifier la soumission érotique comme acte non agentif est problématique); raciste (les femmes jugées capables d'agentivité sexuelle bénéficient généralement des privilèges liés à la blancheur - on n'a qu'à penser aux nombreuses chanteuses africaines-américaines victimes de slut-shaming contrairement à leurs homologues blanches, le slut-shaming étant ici une répression à l'égard d'une trop grande agentivité perçue); et capacitiste (l'agentivité sexuelle vient du fait d'être vue comme une partenaire sexuelle potentielle, ce qui est difficile pour les personnes en situation de handicap puisqu'elles sont systémiquement désexualisées). En fait, dans les définitions actuelles dominantes plutôt psychologisantes, le concept ne tient pas compte du pouvoir structurel qui module nécessairement l'agir sexuel des femmes. Pour la théoricienne crip Abby Wilkerson, l'agentivité sexuelle est justement un élément clé de la lutte pour une justice sociale :

[...] sexual democracy should be recognized as a key political struggle, not only because of the importance of the basic human right to sexual autonomy, but also because (as I will argue) a group's sexual status tends to reflect and reinforce its broader political and social status. I understand sexual agency not merely as the capacity to choose, engage in, or refuse sex acts, but as a more profound good which is in many ways socially based, involving not only a sense of oneself as a sexual being, but also a larger social dimension in which others recognize and respect one's identity (Wilkerson $2002: 35$ ).

Pour Wilkerson, l'agentivité sexuelle est loin d'être un luxe que l'on demande lorsque toutes les autres nécessités sont obtenues, mais constitue plutôt un élément clé ou un baromètre pour comprendre l'oppression. En fait, plus un individu subit de l'oppression liée au genre, la race ou la classe, plus il est susceptible que lui soit retirée aussi son agentivité sexuelle. En ce sens, Wilkerson affirme: "Sexual democracy, then, will require not only opposing the political forces that stigmatize some sexualities as perversions, but also dismantling oppressive social relations including racism, ableism, capitalism, sexism, and ageism which cause some groups' sexuality to be scrutinized in the first place » (Wilkerson $2002: 38$ ).

La question de l'agentivité a été largement débattue dans les sciences sociales et les théories féministes. Il n'est pas question ici de reprendre l'ensemble de ce débat, et nous nous contenterons de dire qu'une posture radicale qui croit au plein libre arbitre des individus ou à un déterminisme pur s'avère difficilement tenable (Emirbrayer et Mische 1998). Une conception de l'agentivité, et à plus forte raison de l'agentivité sexuelle, doit tenir compte des différents facteurs d'oppression: le genre, le sexe, l'orientation sexuelle, la classe, la race, l'ethnicité, l'âge, le handicap ou le manque de 
capital érotique. Ceux-ci ont une influence notable sur les capacités d'agir des femmes dans le domaine de la sexualité.

Les auteures qui ont abordé la question de l'agentivité sexuelle en tenant compte de ces facteurs d'oppression ont souvent basculé du côté déterministe pour n'y voir qu'un effet du système néolibéral. Par exemple, pour Rosalind Gill (2008), l'agentivité est construite à travers la discipline et la régulation du corps pour répondre au système hétéronormatif et patriarcal. Bay-Cheng (2015) propose aussi de voir le concept d'agentivité sexuelle comme une nouvelle forme d'injonction contrôlant la sexualité des jeunes filles. Au contrôle social qu'exerce le continuum de la vierge et de la putain sur la sexualité des filles s'ajoute celui de la démonstration obligatoire de l'agentivité sexuelle. Ainsi, une activité comme l'abstinence se voit évaluée différemment si la personne démontre faire preuve d'agentivité, et pour ce faire, les femmes n'hésitent pas à se désolidariser entre elles pour paraitre plus agentives que d'autres. Pour BayCheng « According to a neoliberal logic, being a victim is not the result of violation by another person, systemic injustice, or even just bad luck; it is the manifestation of one's ineptitude as an agent » (Bay-Cheng 2015 : 286). Chez cette auteure, il ne reste rien de la capacité d'agir, du sentiment d'être à l'origine de ses actes, mais plutôt « the degree of control they proclaim, or are perceived, to exert over their sexual behavior " (BayCheng 2015 : 282). La notion clé ici est le contrôle réel ou perçu qu'une fille démontre dans la sphère sexuelle : l'agentivité devient ainsi une institution par rapport à laquelle l'écart amène une sanction sociale pour ne pas avoir fait preuve de contrôle. Il semble clair qu'il $\mathrm{y}$ a un effet pervers à la transformation des scripts traditionnels où les femmes peuvent faire preuve d'agentivité sexuelle, car il repose maintenant sur les épaules des femmes de montrer que leur sexualité est agentive. Mais considérer l'agentivité sexuelle uniquement comme une norme à atteindre ou de l'autocontrôle sexuel, c'est encore une fois basculer dans une vision purement déterministe qui nie toute agentivité aux femmes, puisque le sentiment d'être à l'origine de ses actes ne serait qu'une fausse conscience produite par le néolibéralisme.

Pour sortir de cette impasse, Jackson et Scott (2010) nous proposent d'appréhender la sexualité selon un modèle à quatre niveaux dans lequel l'agentivité repose sur un soi sexuel réflexif. Le modèle proposé reprend en grande partie les trois niveaux de scripts sexuels de Simon et Gagnon (1973; Simon Gagnon 1986; Gagnon 2008) auxquels il ajoute la dimension structurelle. Ainsi, pour analyser la sexualité, il faut tenir compte de la dimension structurelle que constitue notamment l'institution de l'hétérosexualité; la dimension culturelle qui correspond au scénario culturel de la sexualité ; les pratiques sociales routinières qui correspondent au niveau des scripts interpersonnels; et le soi comme sujet qui construit et interprète les interactions sexuelles. Il s'agit de la vie intrapsychique d'une personne qui inclut, entre autres, l'identité sexuelle, les sentiments de désir et la négociation intérieure avec les normes. Il est important de préciser que ce modèle est circulaire, car il part du structurel pour aller vers l'individu, et vice versa, du micro au macro-social. L'agentivité sexuelle, bien que modulée par les contraintes structurelles, culturelles et les interactions parfois abusives, existe toujours chez chaque sujet. C'est une agentivité qui permet aux femmes de négocier dans ces contraintes et peut-être de transformer les interactions interpersonnelles, les scénarios culturels et, si elles s'allient à d'autres femmes, le structurel. Ainsi, l'agentivité sexuelle ne se construit pas en soi, elle se négocie dans un contexte social et sexuel et doit être reconnue par l'autre comme telle pour devenir une 
réelle agentivité. Ce qui nécessite un contexte sociosexuel propice à son émergence, qui passe notamment par une transformation sociale du script sexuel traditionnel.

Rappelons que, selon Frith et Kitzinger (2001), le script sexuel culturel typiquement masculin s'exprime par la recherche active de partenaires sexuels, l'importance de l'approbation des exploits sexuels par les pairs, une sexualité incontrôlable après excitation et une recherche du plaisir pour le plaisir. Pour les mêmes auteurs, le script sexuel typiquement féminin est caractérisé par l'attente passive d'être choisie comme partenaire sexuelle, un désir plus orienté vers l'affection et l'amour que vers le sexe, l'importance du désir de plaire à l'homme et une absence d'initiative dans les rapprochements sexuels. Il semble évident que sans changement de ce script hétérosexuel traditionnel, il est difficile pour les femmes d'arriver à exercer une agentivité sexuelle. Cependant, on conçoit aussi d'emblée à quel point une part importante des pornographies mainstream proposent des scénarios sexuels en rupture avec ce script sexuel traditionnel : les femmes y sont plus actives et l'affection ainsi que l'amour font rarement partie du scénario, et ce, dès l'âge d'or de la pornographie (Williams 1989). Toutefois, il demeure qu'au sein de bien des pornographies mainstream, on observe des relents de scripts sexuels traditionnels, notamment dans le traitement différentiel de la jouissance féminine où le plaisir des femmes est davantage au service des hommes que l'inverse (le concept d'agentivité sexuelle comprend également le sentiment et l'affirmation d'avoir droit au plaisir pour les femmes).

\section{Méthodologie}

Comme il a été mentionné précédemment, la présente recherche s'inscrit dans un projet plus vaste visant à documenter les caractéristiques de la pornographie identifiée comme féministe et/ou critique. Tout d'abord, nous avons constitué un corpus de films ${ }^{8}$. Ce corpus a été constitué en 2013-2014 et il s'agit d'un corpus non exhaustif, mais qui cherche à être assez représentatif de la diversité de la production de pornographie féministe. Nous avons fait d'abord une revue de la littérature et consulté les Feminist Porn Awards afin d'identifier les productrices majeures. Nous avons cherché à avoir une diversité de genre de films : queer, dyke, trans, BDSM, pour femme, pour couple. Nous voulions aussi avoir les figures plus historiques comme Candida Royalle et Maria Beatty. Il fallait par ailleurs y avoir accès, alors nous avons privilégié les films en ligne disponibles sur Lust Cinéma et choisi une partie des films sur DVD accessibles. Pour être retenus dans ce corpus, les films devaient contenir des représentations explicites de la sexualité et être réalisés par des femmes cis ou des personnes trans s'identifiant comme féministes. Au total, 38 productions ont été retenues, dont 35 longs métrages (le travail de 19 réalisatrices et d'un réalisateur trans) et 3 compilations de courts métrages (30 courts métrages, réalisés par 22 réalisatrices) produits entre 1995 et 2015. Lorsque les DVD comprenaient des entrevues avec les actrices ou réalisatrices ou un making of en supplément (une pratique courante dans la pornographie féministe), ceuxci ont aussi été inclus dans l'analyse. Ensuite, nous avons documenté les différents éléments caractérisant les pornographies critiques. Pour ce faire, l'équipe de recherche a travaillé à l'élaboration d'une grille d'observation qualitative. Cette grille d'observation a été élaborée d'une part en s'appuyant sur la littérature portant sur la pornographie - plus particulièrement les travaux de Williams (1989) -, et d'autre part en s'inspirant des éléments observés de manière non dirigée lors de visionnements de 
productions pornographiques mainstream. Il s'agit donc d'une grille théorique enrichie par les thèmes émergents de l'analyse visuelle. Une première version de la grille d'observation a été testée et bonifiée par l'équipe de recherche suite au visionnement des films pornographiques ne faisant pas partie de l'échantillon. Le visionnement et la rédaction des grilles d'observation ont été effectués par la chercheure principale et une équipe de trois assistantes de recherche durant une période de plusieurs mois. Des rencontres d'équipe périodiques ont permis de mettre en commun les résultats obtenus et de peaufiner la compréhension des différentes catégories d'analyse de la grille, s'assurant du fait même d'un accord interjuge.

16 La version définitive de la grille contient une quarantaine d'indicateurs répartis en cinq grandes catégories soit : les destinataires, le film comme produit esthétique et culturel (trame sonore, montage, plans de caméra, etc.), la trame narrative (ce qui pousse les personnages à avoir des relations sexuelles, les fonctions de la scène sexuelle par rapport à la trame narrative, etc.), les discours politiques explicites ou sous-jacents (discours sur le féminisme, le travail du sexe, l'inclusion de personnes minorisées, etc.), et finalement la catégorie structures/scripts des sexualités représentées (relations entre les partenaires sexuels, expression du consentement sexuel, numéros sexuels représentés, etc.). Pour cet article, nous avons concentré notre analyse sur la dernière catégorie - structures/scripts des sexualités représentées - qui est utilisée pour faire l'analyse plus spécifique de la représentation de l'agentivité sexuelle dans l'échantillon. Cette dernière catégorie de la grille comporte, entre autres, l'observation de l'expression du consentement, des préférences sexuelles ainsi que la remise en question du script hétérosexuel traditionnel. Ces trois éléments constituent les marqueurs que nous avons identifiés et choisi de mettre en avant afin de rendre compte de l'agentivité sexuelle des femmes dans notre corpus.

17 Le premier marqueur, soit l'expression du consentement, réfère généralement à la communication verbale ou non verbale, libre de toute contrainte, de sa volonté à entreprendre une activité sexuelle donnée. Le consentement sexuel peut être retiré en tout temps. Le deuxième marqueur, l'expression des préférences sexuelles, réfère au fait de communiquer à son/sa/ses partenaire(s) ses envies sexuelles concernant toutes les dimensions de l'expérience sexuelle (comment toucher et être touchée, comment se parler, etc.). L'expression des préférences sexuelles implique la reconnaissance d'un droit au plaisir et à poser une action afin de combler un désir. Le troisième marqueur est plus complexe, il s'agit du conformisme, de la transformation ou de la remise en question du script hétérosexuel traditionnel. La déconstruction ou la critique du script hétérosexuel traditionnel constitue selon nous la condition de possibilité de l'agentivité sexuelle.

\section{Résultats}

\section{Le consentement : la condition initiale de l'agentivité sexuelle}

18 Afin de parler de la représentation de l'agentivité sexuelle des femmes, il faut minimalement qu'un consentement sexuel soit accordé par les actrices ou les personnages des films analysés. Nous choisissons d'utiliser le terme actrice ou personnage, car la pornographie est un médium qui entretient un rapport particulièrement complexe au réel (Benjamin 1995) : les actes sexuels mis en scène sont 
réellement performés bien que la forme, la mise en scène, l'enchainement, les actes sexuels puissent être dictés par une tierce personne (la réalisatrice). De plus, dans plusieurs films, les actrices jouent plus ou moins leur propre rôle ou dans d'autres cas, les actrices sont interviewées dans le film ou dans le making of. Dans notre échantillon, le consentement ou son absence s'exprime selon six formes : implicite, explicite, par le biais du dirty talk, inscrit dans la diégèse, le non-consentement explicite respecté et le plus problématique, le non-consentement non respecté (il s'agit alors de violence sexuelle). Nous avons classé les patrons de consentement du plus fréquemment au moins fréquemment observé, et nous avons segmenté les divers numéros sexuels plutôt que les films, car, dans certains films comme The Band, les formes de consentement sont variables, parfois idéales et parfois problématiques selon une perspective féministe.

La forme la plus fréquente est définitivement le consentement implicite. Il s'exprime par le langage non verbal ou semble être chuchoté. Par exemple, dans le film Matinée de Lyon Bell, le consentement transparait dans les échanges de regards intenses entre les personnages, les hochements de tête, la lenteur des gestes. Cette manière de donner le consentement pose problème pour la personne qui analyse, car il doit être inféré par l'enthousiasme que se témoignent les partenaires, par leur complicité, par le langage non verbal significatif (par exemple, tirer le partenaire vers soi) ou par le hochement de tête.

Ensuite, vient le consentement exprimé de manière explicite. Notamment dans le film de Courtney Trouble, Come find me, où tout au long de la relation entre les partenaires hétérosexuels plusieurs questions sont posées comme « do you want me to... pinch you/ spank you/tease you ", et une réponse est chaque fois donnée par l'autre partenaire. Il ne s'agit plus d'un consentement à une relation sexuelle dans son ensemble, mais bien d'une négociation de tous les instants. L'autre exemple intéressant de ce type de consentement explicite se trouve dans Silver Shoes de Jennifer Lyon Bell. Dans la scène lesbienne, Liandra Dahl demande souvent à sa partenaire si elle veut telle caresse, si elle aime telle pratique, si elle peut prendre un jouet, et ce, à plusieurs occasions. Les films BDSM du corpus sont particulièrement intéressants aussi à l'égard du consentement explicite. Dans le film 50 Shades of Dylan Ryan de Madison Young, le consentement entre Mme Grey et Dylan est d'abord établi lors de la négociation du pari ${ }^{9}$, celui de Brenda est réitéré verbalement par des remerciements fréquents à Mme Grey du type "Oh oui! J'aime ça! Merci, merci beaucoup Mme Grey!» (notre traduction) et le consentement de Dylan est réitéré même hors des scènes sexuelles, notamment lorsqu'elle tourne ses capsules vidéos.

21 Le troisième patron de consentement passe par le dirty talk. Par exemple, dans le film Bordello de Courtney Trouble, le consentement est souvent donné spontanément ou en réponse à une question du partenaire par le biais de mots coquins (par exemple : «Fuck me like that, go faster » ou «I want you to fuck me. - Want me to fuck you hard? Really hard.»), alors que dans le making of du film, toutes les limites des actrices sont établies, ce qui nous renseigne non seulement sur le consentement à avoir une relation sexuelle, mais également que toutes les activités sexuelles sont préalablement consenties. Le dirty talk devient ainsi une manière sexy ou «théâtrale » de mettre en scène le consentement dans la représentation fictionnelle.

Le quatrième patron de consentement est intégré à la diégèse. L'exemple le plus significatif du corpus se trouve dans Doing it ourselves, the trans women porn project de Tobi Hill-Meyer. L'histoire du film repose sur le désir de plusieurs femmes trans de 
réaliser un film à l'image de leurs sexualités. Ainsi, les transitions entre les numéros sexuels sont remplies de dialogues sur le désir de participer au film, leur souhait d'avoir des relations sexuelles ensemble, dans tel décor : ces dialogues font avancer l'histoire de la création du film. De même, Fluid: Women Redefining Sexuality de Madison Young se construit comme un documentaire pornographique et intercale des entrevues avec les actrices et les scènes sexuelles qui imagent les propos de l'interviewée. Dans ces entrevues, les actrices relatent leur parcours identitaire, leur point de vue sur le travail du sexe, mais aussi les actes et caresses qu'elles préfèrent lorsqu'elles ont des relations sexuelles, et ce sont ces mêmes éléments que l'on retrouve mis en scène dans le numéro sexuel qui suit. Dans cette structure filmique, les entrevues ont plus qu'une fonction de making of, elles ont une fonction narrative, elles font avancer l'histoire, non pas fictive, mais documentaire.

Le cinquième n'est pas un patron de consentement, mais plutôt un patron de refus ou de consentement illustré à la négative. Dans All about Anna de Jessica Nilsson, Anna verbalise son absence de consentement à plusieurs reprises à un partenaire, et finit par serrer fermement son pénis pour lui faire mal et pour lui signifier son refus. La scène se termine ainsi et sans réplique du partenaire.

Finalement, il y a quelques scènes où le consentement est non respecté au sein de la narration, notamment dans deux mises en scène claires de viol. La première scène se trouve dans Cabaret désir d'Erika Lust où la protagoniste, une cambrioleuse professionnelle, viole un homme afin de pouvoir avoir un enfant. L'histoire est racontée par l'enfant issu du viol devenu adulte, qu'il présente comme le chef-d'œuvre de la carrière de cambrioleuse de sa mère. Dans la scène elle-même, l'homme violé, qui une fois la surprise surmontée est présenté comme appréciant grandement son expérience sexuelle, n'est pas sans rappeler un leitmotiv fréquent dans certaines pornographies mainstream, les rôles genrés étant cette fois-ci renversés. L'autre occurrence de viol, dans Oooooh! de Sophie Bramly, est plus singulière (toutes pornographies confondues). Le film raconte l'histoire d'un couple dont la femme désire améliorer sa vie sexuelle (elle n'a jamais eu d'orgasme) en inscrivant son couple à une formation sexuelle pratique en pension complète en guise de cadeau surprise d'anniversaire à son conjoint. Lorsqu'il se rend compte de l'objet du séjour, il réagit très mal et viole sa femme. La mise en scène du viol n'est pas transformée en numéro sexuel classique où la femme serait finalement très enthousiaste, mais reste une représentation de viol où le conjoint contraint sa femme qu'il prend par derrière, déchire ses collants et la pénètre violemment pendant qu'il l'insulte. La femme ne réagit aucunement, sa tête enfouie dans un oreiller est maintenue fermement par le conjoint.

\section{Communications érotiques des préférences sexuelles}

Notre deuxième marqueur d'agentivité sexuelle réside dans l'expression des préférences sexuelles. Dans les films de notre corpus, les préférences sexuelles sont exprimées verbalement (dans le film ou dans les entrevues qui accompagnent le film) ou non verbalement. Dans 19 films sur 38, les préférences sexuelles des femmes sont exprimées verbalement. L'expression des préférences des partenaires n'est pas toujours constante dans les films. Dans des films comme Bordello, Trans Grrrls, Come find me de Courtney Trouble ou encore, Silver Shoes de Lyon Bell, l'expression des préférences est plutôt fréquente, voire pour chaque pratique sexuelle, alors que dans des films comme 
Eyes of Desire 1 et 2, Billy Castro does the mission ou Taxi, il n'y a que quelques occurrences d'expression verbale. Par exemple, Dylan Ryan explique dans Fluid: Women redefining sexuality, les techniques précises de fisting qu'elle souhaite recevoir et qui lui permettront d'éjaculer. Dans Billy Castro does the mission, Ryan demande directement un cunnilingus à un moment précis de la relation sexuelle. Plusieurs préférences sont aussi présentées dans certains films par l'intégration d'entrevues avec les actrices. Dans Expert guide to anal pleasure for men, Fluid: Women redefining sexuality ou Fluid: Men redefining sexuality, plusieurs acteurs et actrices parlent de leurs préférences sexuelles précises dans les entrevues qui ponctuent les films. Dans d'autres cas, les préférences sont discutées en amont du tournage et présentées comme gage d'« authenticité » dans le making of souvent inclus dans les DVD. Comme pour le consentement, certaines préférences sexuelles sont aussi exprimées sous la forme de dirty talk. Les préférences peuvent aussi être exprimées non verbalement, en guidant les gestes du ou de la partenaire. Dans Matinée, l'actrice repositionne clairement la main de l'acteur lorsqu'il la masturbe. Une autre manière plus implicite d'exprimer les préférences, c'est à travers la manifestation du plaisir, de la satisfaction à travers des mouvements du corps, des soupirs. Ces manifestations du plaisir peuvent être lues comme une incitation à continuer la stimulation ou une connaissance préalable de la préférence de l'autre.

\section{Transformation ou remise en question du script hétérosexuel traditionnel}

Pour faire état de ce dernier marqueur, nous avons décidé de séparer le corpus en deux : d'un côté, les films présentant des sexualités lesbiennes ${ }^{10}, \mathrm{BDSM}$, queer ou impliquant des personnes trans (17 des 38 films); de l'autre, les films présentant des sexualités hétérosexuelles ou bisexuelles ( 22 des 38 films ${ }^{11}$ ).

Dans les films lesbiens, queer, BDSM ou trans, il y a nécessairement une remise en question du script hétérosexuel traditionnel. Bien que dans certains cas de relations butch/fem, le script traditionnel soit maintenu, celui-ci expose d'emblée son caractère construit, voire factice, puisque l'hétérosexualité est explicitement performée. Plus précisément, l'analyse de notre corpus nous a permis d'observer que les scripts sont transformés de cinq manières.

La première s'exprime par la centralité du plaisir des femmes cis et personnes trans, et ce, dans la totalité des 17 films de ce sous-groupe. Par exemple, le film de Hill-Meyer Doing it ourselves, the trans women porn project, est politiquement motivé par l'absence de pornographie représentant la sexualité des femmes trans. En fait, pour Hill-Meyer, la pornographie commerciale qui inclut des corps trans tombe souvent dans la niche de la pornographie shemale qui ne s'intéresse pas à la sexualité des femmes trans, mais aux fantasmes des hommes qui souhaitent des femmes très féminines ayant un gros pénis en érection. Le film de Hill-Meyer repose effectivement sur la pluralité de la sexualité et les plaisirs des femmes trans.

La deuxième manière dont les réalisatrices du corpus transforment le script hétérosexuel traditionnel s'actualise par un travail explicite sur le genre. Si les scripts de genre sont transformés ou conscientisés dans un dessein de subversion, les scripts hétérosexuels traditionnels seront nécessairement bousculés, car ceux-ci se fondent sur des scripts de genre tout aussi traditionnels (Gagnon, 2008). Dans 13 films sur les 17 
qui composent ce sous-groupe, le genre est queerisé. Une minorité de femmes cis hyperféminines et à fortes poitrines est assez notable dans cette sous-section du corpus. La figure de la butch dans toutes ses déclinaisons plus ou moins masculines occupe une place importante, souvent dans des relations butch/fem, mais aussi butch/ butch comme dans Crash pad. Sa présence constitue un élément essentiel permettant de démarquer la pornographie lesbienne du "girl-on-girl» issu des pornographies mainstream (Butler H., 2004, 2015). On retrouve aussi un travail conscient sur la féminité. L'exemple le plus éloquent est Fluid: Women redefining sexuality car les actrices explicitent dans les entrevues qui ponctuent le film leurs parcours identitaires sexuels et de genre. Par exemple, l'actrice Aiden Starr explique que, bien qu'elle soit une femme, elle n'aime pas les étiquettes et vit son genre psychologique comme étant « très ambigu et androgyne » (notre traduction). Elle mentionne que le plaisir qu'elle retire à porter des talons hauts ne provient pas du fait qu'ils contribuent à la faire se sentir féminine, mais bien du fait qu'ils ont un potentiel de fétichisation et qu'ils peuvent blesser (allusion à sa sexualité sadomasochiste). En fin d'entrevue, elle rapporte qu'elle aime avoir un pénis et porter des gode-ceintures en plus de faire un retour sur son androgynie: "I live in between male and female. Sometimes when I wake up I dress like a boy, and act like a boy, and sometimes when I wake up, I dress like a girl and act like a girl ».

La troisième manière de transformer le script hétérosexuel traditionnel s'actualise par des rapports de pouvoir explicitement performés. L'exemple le plus explicite se déroule dans les films qui présentent une sexualité BDSM où les positions de pouvoir sont négociées entre les partenaires et ne reflètent pas les situations de domination que l'on retrouve dans un système hétéropatriarcal et raciste. Déjà, les trois films et le court métrage BDSM que nous avons sélectionnés (deux films de Maria Beatty, un film de Madison Young et le court métrage Authority de Marit Östberg compilé dans Dirty diaries. 12 shorts of feminist porn) sont entièrement composés de femmes. Dans The Black Glove de Maria Beatty, la soumise est également la cinéaste. Ainsi, bien qu'elle soit sous la domination des Maîtresses Morgana ou Sabrina, elle a elle-même établi les règles du scénario érotique et en est donc la réelle décideuse.

31 La quatrième manière de transformer le script hétérosexuel traditionnel dans les films $\mathrm{du}$ corpus passe par une désessentialisation des rôles passifs et actifs dans la pénétration ou la fellation par l'emploi du gode-ceinture. Que le gode-ceinture soit porté par une femme cis féminine ou masculine, une femme ou un homme trans, pour pénétrer une femme cis féminine ou masculine, une femme ou un homme trans, tous les scénarios ont été proposés et traduisent un désir de désessentialiser la sexualité en utilisant un objet culturel pour la pénétration. Le gode-ceinture permet non seulement de révéler le caractère construit de la sexualité, mais de subvertir les rôles sexuels de manière explicite, ou du moins d'exposer le caractère performatif de ceux-ci. Le scénario est plus explicitement subversif dans les films de Buck Angel : comme homme trans, il est tour à tour pénétrant et pénétré avec un gode-ceinture. Dans une majorité de cas, les femmes plus masculines pénètrent les femmes plus soumises ou féminines, révélant l'aspect construit du genre tout en reprenant les scripts de genre plus classiques. Par exemple, le film Nostalgia illustre une reprise des classiques de la pornographie comme Deep throat, mais uniquement avec des femmes qui portent des godes-ceintures, où même le deep throathing est réalisé sur un gode et où l'éjaculation est simulée par une substance contenant des paillettes (squirting glitter). 

plus spécifique au genre pornographique (tel que théorisé par Williams 1989). En effet, dans les pornographies mainstream chaque numéro sexuel se termine par une éjaculation pénienne externe, couramment nommée "money shot ». Le money shot est d'une importance centrale, car il marque la fin du scénario sexuel en plus d'authentifier la véracité de l'orgasme (Williams 1989). Le script pornographique se termine presque toujours par cette scène d'éjaculation masculine. Or, dans 10 films sur les 17 que compte notre sous-groupe, le money shot est remplacé par l'éjaculation féminine. Ce money shot féminisé possède la même fonction narrative de clore les numéros sexuels, mais permet par la même occasion de décentrer le script de l'orgasme masculin. Il faut dire qu'il y a une absence notable d'hommes cis dans cette partie du corpus. hétérosexuel traditionnel est également transformé. Nous avons pu observer quatre divergences notables. En premier lieu, comme pour le sous-groupe queer et lesbien, le plaisir et l'orgasme féminins sont centraux dans presque tous les films (21 films sur 22 dans ce sous-groupe), contrairement aux scripts des films mainstream. Non seulement les femmes constituent les personnages centraux des films du corpus, mais leur quête de plaisir sexuel s'avère souvent l'intrigue principale. Dans certains films comme Dude looks like a lady d'Erika Lust ou Stud hunters de Candida Royalle, on compte davantage d'orgasmes féminins que masculins représentés. Dans Oooooh!, on suit littéralement la quête orgasmique d'une femme qui, contrairement à Deep throat où cette même quête s'avère une excuse pour présenter un maximum de fellations, est réellement centrée sur une éducation sexuelle visant l'orgasme et la maximisation du plaisir de la protagoniste. rapports sexuels et faire progresser le déroulement des actes sexuels, un scénario moins commun dans toutes les pornographies tant féministes que mainstream (Gagnon 2008). Dans notre corpus, ce sont les femmes qui initient et qui ont un rôle actif dans la majorité des films, soit dans 17 films sur 22. Encore ici, l'exemple de Dude looks like a lady est assez éloquent, car la femme est en contrôle : elle maquille l'homme, lui met une perruque, lui fait porter une culotte et une robe ; elle dirige tout le rituel, et il fait tout ce qu'elle demande, passivement. De même, dans Matinée de Lyon Bell, c'est la protagoniste qui initie la scène, les changements de position et qui dirige l'action. Même lorsqu'elle reçoit du plaisir, elle garde un certain contrôle en donnant des indications à son partenaire. Elle prend les décisions, l'autre l'accompagne.

ous avons aussi constaté l'inclusion de représentations où l'homme est pénétré. Dans cinq films du corpus présentant une sexualité hétérosexuelle, l'homme se trouve ainsi pénétré par sa partenaire. Il s'agit du motif central du film de Taormino, Expert guide to anal pleasure for men, un film éducationnel sur le pegging et les diverses stimulations anales de la prostate. Dirty diaries, un des courts métrages, met aussi en scène une femme qui pénètre l'anus de son partenaire avec un doigt sans que le film porte sur cette pratique spécifique comme dans les deux autres exemples.

Finalement, dans quelques films ( 5 sur 22), la sexualité continue après l'éjaculation masculine. Dans le script hétérosexuel traditionnel, l'acte sexuel se termine toujours par l'orgasme de l'homme et dans les différentes pornographies hardcore, cet orgasme est signifié par une scène d'éjaculation externe. Cette transformation du script 
témoigne précisément que le plaisir sexuel masculin n'est pas le point culminant de la sexualité comme c'est le cas dans la pornographie mainstream. Dans Come find me, l'orgasme de l'homme sera suivi d'un cunnilingus, un écart plutôt inusité par rapport au script traditionnel dans l'industrie pornographique (Williams 1989).

\section{Discussion}

37 L'analyse de la représentation de la sexualité du corpus corrobore les résultats de la recherche de Liberman (2015) auprès des productrices, actrices et consommatrices de pornographie féministe. Notre analyse confirme effectivement que la pornographie féministe est un espace hétérotopique où cohabitent différentes positions sur la sexualité, notamment au sujet du consentement, et qui offre des représentations de femmes agentives sexuellement. De même, le constat d'Attwood (2011) selon lequel on assiste au passage de l'objectivation sexuelle à un mode de présentation du soi sexuel performatif dans les nouvelles pratiques de webcam et les pornographies alternatives comme SuicideGirls ou Furry Girls, converge avec les films de notre corpus qui présentent des entrevues avec les actrices et productrices. Doing it ourselves, the trans women porn project ou Fluid: Women redefining sexuality explicitent une présentation du soi sexuel imbriquée à même le film, tandis qu'une majorité de films queer le font plutôt dans le making of. Dans d'autres cas, on peut inférer à partir d'entrevues insérées dans d'autres films qu'il s'agit d'une présentation du soi sexuel : par exemple, Dylan Ryan dans Billy Castro does the mission, présente une sexualité et un personnage qui véhiculent ses idées politiques, ses valeurs sexuelles et sa manière sexuelle d'être à l'écran, qu'elle explicite dans Fluid: Women redefining sexuality.

L'analyse des films composant notre corpus nous aura permis de confirmer la présence accrue de représentations de femmes sexuellement agentives dans la pornographie féministe. Plus spécifiquement, les films de notre corpus mettent en avant des représentations diversifiées et majoritairement claires du consentement sexuel. L'étude du consentement dans notre corpus a aussi fait apparaître un résultat inattendu. Dans une dizaine de films, la représentation du consentement est problématique dans la narration. On constate la présence de scènes ambigües où certains comportements peuvent être interprétés comme des agressions ou du harcèlement. Dans Cabaret désir, une femme viole un homme pour procréer sans qu'aucune conséquence négative n'y soit associée et l'homme agressé y prend plaisir. Dans Ooooh!, la scène d'agression sexuelle n'est pas présentée comme un numéro sexuel classique où la contrainte sexuelle se transforme en relation sexuelle enthousiaste où les deux partenaires prennent plaisir à l'échange. Cependant, on n'assiste pas non plus à une condamnation de la scène de viol : la conjointe passera simplement l'éponge sur cette agression et il n'y a aucune inclusion de conséquence envers le mari à la suite de cet événement dans la trame narrative. Ce résultat du non-consentement sexuel est inattendu d'un point de vue féministe. Par contre, il n'est pas étonnant de retrouver le scénario de sexualité contrainte compte tenu de la prévalence de ce dernier dans tous types de pornographie, y compris dans l'imaginaire de nombreuses femmes (Larsson 2017). Il faut rappeler que les différentes pornographies présentent une sexualité qui vise l'excitation et relèvent souvent du fantasme (dans le sens psychanalytique) que l'on ne souhaite pas nécessairement réaliser. La recherche de Ciclitira (2004) ainsi que les travaux plus théoriques de Benjamin (1995) et Butler J. (1990b) traitent justement de 
cette disjonction des affects entre ce qui est vécu comme sexuellement excitant et notre perception de ce qui devrait être une représentation juste d'une sexualité féministe. Benjamin parlera de ce sentiment, théorisé par Kristeva (1980), d'être étranger à soi-même à l'égard de la pornographie. Ce qui complexifie aussi notre analyse, c'est que les films qui incluent des représentations de sexualité contrainte vont souvent aussi contenir des représentations claires du consentement dans d'autres scènes. Certains films restent assez exemplaires, comme come find me où le consentement est demandé explicitement à presque chaque étape, mais la très grande majorité des films peinent à inscrire systématiquement le consentement verbal. Les moyens identifiés pour assurer le consentement sont plus souvent extradiégétiques (making of, entrevues). Le dirty talk semble une voie couramment suivie pour érotiser ou théâtraliser le consentement dans notre corpus.

Dans une majorité de cas, les femmes sont en mesure de dire ce qu'elles désirent sexuellement (en entrevue ou dans le scénario), se donnant ainsi le droit au plaisir. Certaines iront aussi jusqu'à guider par des gestes ou des indications claires le, la ou les partenaire(s) dans le but d'accroître leur plaisir ou de les mener à la jouissance. Que les préférences soient communiquées verbalement ou non témoigne du fait que les actrices s'accordent le droit au plaisir et qu'elles agissent concrètement pour combler leur désir. Plus indirectement, lorsque l'actrice exécute un scénario qui n'est pas le sien (dans le cas où les numéros sexuels sont orchestrés par la réalisatrice), on assiste à la mise en scène de scripts sexuels novateurs où les femmes prennent en main leur sexualité et transmettent leurs préférences sexuelles sans devoir subir de conséquences négatives. La capacité à exprimer ses préférences sexuelles et à se donner le droit au plaisir constituent en fait deux composantes parmi les plus exigeantes du concept d'agentivité sexuelle. Le fait que les films mettent en scènes un tel niveau d'agentivité s'avère prometteur pour concurrencer les scénarios sexuels traditionnels.

De plus, il est clair que dans tous les films, le plaisir des femmes cis ou des personnes trans, bisexuelles, lesbiennes, pansexuelles, queer, ou hétérosexuelles, dominantes ou soumises, est centrale. La représentation de l'orgasme et du plaisir est centrée sur les femmes, sans non plus détrôner complètement la scène du money shot comme signifiant privilégié pour signaler la fin de l'acte sexuel et l'authenticité de la jouissance (Williams 1989). Même dans des films comme Dude looks like a lady, où la protagoniste contrôle toute la mise en scène sexuelle et où ses orgasmes sont plus nombreux, le marqueur final de l'acte sexuel demeure le money shot du partenaire masculin. Les films lesbiens, queer et trans de notre corpus utiliseront l'éjaculation féminine comme signifiant de l'orgasme, d'une part, parce qu'il n'y a pas d'hommes cisgenres dans les films, et d'autre part, par souci explicite de transformer, de queeriser le script hétérosexuel traditionnel et de visibiliser l'éjaculation féminine, une démarche déjà bien amorcée par Annie Sprinkle au début des années 1990 (Lavigne 2014). Dans les films comprenant des hommes cisgenres, seulement 5 films sur les 17 ne représentent pas le money shot: l'orgasme de l'homme est interrompu par des raisons diégétiques extrasexuelles ou simplement signifié autrement. Le fait que dans plusieurs films l'éjaculation masculine externe ne signifie pas la fin de l'acte sexuel constitue peut-être le résultat le plus intéressant de notre analyse. En fait, lorsque la relation sexuelle continue après l'éjaculation, cela a pour conséquence : d'une part, de resignifier le money shot comme un signifiant quelconque et non plus comme le signifiant par excellence ; et d'autre 
part, de remettre en question le primat de la jouissance masculine dans la sexualité pornographique.

41 Finalement, les rôles sexuels et de genres sont retravaillés, subvertis et parodiés dans les scripts sexuels mis en scène dans les films du corpus. Dans tous ces films, les femmes prennent une part active dans l'initiation des actes sexuels, elles mènent les différentes étapes et dans plusieurs cas, pénètrent aussi leurs partenaires, femmes ou hommes, cis ou trans. Les femmes cis et trans peuvent être dominantes, soumises, butch, fem. Cette diversité permet de décrisper les rôles attribués à chaque genre dans les scripts sexuels hétérosexuels traditionnels. Ainsi, les femmes peuvent démontrer une agentivité claire sans représailles. En fait, le travail sur le genre effectué de manière plus soutenue dans les films queer constitue un effort politique de déconstruire la sexualité et subvertir ou jouer avec les normes de genre associées à la sexualité. Par ailleurs, la présence importante de jouets sexuels, en particulier le godeceinture, indique un désir de désessentialiser la sexualité et les rôles de genre. L'utilisation de ces jouets participe à déconstruire une vision essentialiste de la sexualité qui repose sur le socle de l'instinct de procréation, soit une sexualité hétérosexuelle centrée sur la pénétration phallovaginale. En révélant explicitement le caractère construit de la sexualité et du genre, une renégociation ou une critique peut plus facilement advenir.

En somme, tous ces éléments nous permettent d'affirmer que la présence marquée de représentations de l'agentivité sexuelle des femmes constitue un élément important qui caractérise les pornographies féministes. Cependant, comme il s'agit d'une pornographie encore marginale, les scénarios proposés demeurent eux aussi assez marginaux et plus ou moins accessibles au grand public. Toutefois, l'apport de la pornographie féministe s'avère incontournable. Bien qu'il s'agisse de représentations d'interactions sexuelles, ces pornographies sont également des scénarios culturels qui ouvrent les conditions de possibilité du vécu sexuel des femmes. En reprenant le modèle quaternaire de Jackson et Scott (2010), ces pornographies débutent avec les scripts intrapsychiques des réalisatrices ou des actrices, qui seront négociés entre partenaires dans le film, pour finalement être constitués en produit culturel par la réalisatrice. Inversement, ces produits culturels diffusent des scénarios culturels divergents qui entrent en compétitions avec une diversité de scénarios culturels, des scripts interpersonnels (expériences) et les fantasmes idiosyncratiques, influençant donc indirectement les femmes dans leur vécu sexuel en élargissant la banque de scénarios disponibles. De même, les films plus queer remettent directement en question le sexisme, l'hétérosexisme et la cisnormativité et agissent directement sur le niveau structurel. Ainsi, la pornographie féministe joue sur les trois niveaux de scripts sexuels et tente de changer la sexualité à un niveau structurel. La plus-value de la représentation de l'agentivité sexuelle des femmes dans la pornographie féministe, c'est qu'elle permet d'élargir le répertoire sexuel en changeant les scripts sexuels disponibles: les scripts sexuels représentés proposent des exemples d'agentivité sexuelle exempte de slut-shaming, ce qui contribue à modifier les conditions de possibilité du vécu sexuel des femmes, leur permettant d'explorer et de s'approprier plus librement une diversité sexuelle. 


\section{BIBLIOGRAPHIE}

ATTWOOD, Feona. 2007. « Sluts and riot grrrls: Female identity and sexual agency » Journal of Gender Studies, 16(3) : 233-247.

ATTWOOD, Feona. 2011. « Through the looking glass? Sexual agency and subjectification online » in New femininities, GILL, Rosalind \& SCHARFF, Christina (éd.). UK: Palgrave Macmillan, 203-214.

BAY-CHENG, Laina Y. 2015. « The agency line: A neoliberal metric for appraising young women's sexuality » Sex Roles, 73: 279-291.

BENJAMIN, Jessica. 1995. « Sympathy for the Devil: Notes on Sexuality and Aggression » in Like subjects, love objects: Essays on recognition and sexual difference. New Haven et Londres: Yale University Press, 175-221.

BRIDGES, Anna. J., SUN, Chyng. F., EZZELL, Matthew. B. \& JOHNSON, Jennifer. 2016. « Sexual Scripts and the Sexual Behavior of Men and Women Who Use Pornography » Sexualization, Media, \& Society [En ligne], 2(4), consulté le 20 mai 2019. URL : https://journals.sagepub.com/doi/full/ $10.1177 / 2374623816668275$; DOI :10.1177/2374623816668275.

BUTLER, Heather. 2004. « What do you call a lesbian with long fingers? The development of dyke pornography » in Porn Studies, WILLIAMS, Linda (éd.). Durham: Duke University Press, 167-197.

BUTLER, Heather. 2015. «Que dit-on d'une lesbienne aux doigts longs » in Cultures pornographiques. Anthologie des porn studies, VÖRÖs, Florian (éd.). Paris : Amsterdam, 161-193.

BUTLER, Judith. 2006 [1990a]. Trouble dans le genre : Le féminisme et la subversion de l'identité. Paris : La Découverte.

BUTLER, Judith. 1990b. « The Force of Fantasy: Feminism, Mapplethorpe, and Discursive Excess » Differences: A Journal of Feminist Cultural Studies, 2(2): 105-125.

CORSIANOS, Marilyn. 2007. « Mainstream pornography and "Women": Questioning sexual agency » Critical Sociology, 33(5-6): 863-885.

BEAUVOIR, Simone de. 1949. Le deuxième sexe. Paris : Gallimard.

LAURETIS, Teresa de. 1987. Technologies of Gender: Essays on Theory, Film, and Fiction. Bloomington and Indianapolis: Indiana University Press.

DINES, Gail. 2010. Pornland: how porn has hijacked our sexuality. Boston, Mass.: Beacon Press.

EMIRBRAYER, Mustafa \& MISCHE, Ann. 1998. « What is agency? » American Journal of Sociology, 103: 962-1023.

FETTEROLF, Janell. C. \& SANCHEZ, Diana T. 2015. « The costs and benefits of perceived sexual agency for men and women » Archives of Sexual Behavior, 44(4): 961-970.

FOUCAULT, Michel. 1976. Histoire de la sexualité : La volonté de savoir. Paris : Gallimard.

FRITH, Hannah \& KITZINGER, Celia. 2001. « Reformulating sexual script theory: Developing a discursive psychology of sexual negotiation » Theory \& Psychology, 11(2): 209-232.

GAGNON, John H. \& WILLIAM, Simon. 1973. Sexual conduct: The social sources of human sexuality. Chicago: Aldine Pub. Co.

GAGNON, John H. 2008. Les scripts de la sexualité. Paris : Payot. 
HALL, Stuart. 1980. « Encoding/decoding » in Culture, media, language. Working Papers in Cultural Studies, 1972-1979, Centre for Contemporary Cultural Studies (éd.). London: Routledge, 128-138.

HILL-MEYER, Tobi. 2013. « Where the trans women aren't: The slow inclusion of trans women in feminist and queer porn » in The feminist porn book: The politics of producing pleasure. TAORMINO, Tristan, SHIMIZU, Celine Parreñas, PENLEY, Constance \& MILLER-YOUNG, Mireille (éd.). New York: Feminist Press at the City University of New York, 155-163.

HORNE, Sharon \& ZIMMER-GEMBECK, Melanie J. 2005. « Female sexual subjectivity and wellbeing: Comparing late adolescents with different sexual experiences » Sexuality Research and Social Policy, 2(3): 25-40.

KIM, Janna. L. 2007. «From Sex to Sexuality: Exposing the Heterosexual Script on Primetime Network Television » Journal of Sex Research, 44(2): 145-157.

KIRSCH, Alexandra. C. \& MURNEN, Sarah. K. 2015. «"Hot” girls and "cool dudes": Examining the prevalence of the heterosexual script in American children's television media » Psychology of Popular Media Culture, 4(1): 18-30.

JACKSON, Stevi \& SCOTT, Sue. 2010. Theorizing sexuality. Berkshire, England: McGraw-Hill.

LANG, Marie-Ève. 2011. « L“"agentivité sexuelle” des adolescentes et des jeunes femmes : Une définition » Recherches féministes, 24(2) : 189-209.

LARSSON, Maria. 2017. « Lasse Braun, rape scenarios, and Swedish censorship: a case study of two 8-mm porn films featuring rape » Porn Studies [En ligne], 4(1) : 23-34, consulté le 29 mai 2019. URL : https://www.tandfonline.com/doi/full/10.1080/23268743.2016.1240949 ; DOI : 10.1080/23268743.2016.1240949.

LAVIGNE, Julie. 2014. « La post-pornographie comme art féministe : la sexualité explicite de Carolee Schneemann, d'Annie Sprinkle et d'Émilie Jouvet » Recherches féministes, 27(2) : 63-79.

LAVIGNE, Julie. 2009. « Sexualité et photographie : Transgression féministe et ratification de la norme pornographique comme pratique artistique » Protée. Revue internationale de théories et des pratiques sémiotiques, 37(1) : 25-34.

LE BLANC ÉLIE, Myriam, LAVIGNE, Julie \& MAIORANO, Sabrina. 2017. « Cartographie des pornographies critiques » Genre, sexualité \& société [En ligne], 17(17) : 1-24. Consulté le 20 mai 2019. URL : https://journals.openedition.org/gss/4007 ; DOI :10.4000/gss.4007.

LAVIGNE, Julie. 2017. « Autopornography and the struggle for the recognition of a sexual subjectivity: A theoretical analysis from Loree Erickson's testimony in The Feminist Porn Book » Feminist Media Studies. 17(5): 790-803.

LEMONCHECK, Linda. 2010. Loose women, lecherous men: A feminist philosophy of sex. New York: Oxford University Press.

LERUM, Kari \& DWORKIN, Shari. L. 2015. «Sexual agency is not a problem of neoliberalism: Feminism, sexual justice, \& the carceral turn » Sex Roles, 73: 319-331.

LIBERMAN, Rachael. 2015. « "It's a really great tool”: Feminist pornography and the promotion of sexual subjectivity » Porn Studies, 2: 174-191.

MACKINNON Catharine A. 1982. « Feminism, Marxism, Method, and the State: An Agenda for Theory » Signs, 7 (3): 515-544.

MAINA, Giovanna. 2014. «Belladonna's strapped dykes between mainstream and queer » in Porn after porn: contemporary alternative pornographies, BIASIN, Erico, MAINA, Giovanna \& ZECCA, Federico (éd.), Milano: Mimesis International, 83-106. 
MAXWELL, Claire \& AGGLETON, Peter. 2010. « Agency in action: Young women and their sexual relationships in a private school » Gender and Education, 22(3): 327-343.

MCCABE, Janet \& AKASS, Kim. 2007. Quality TV: Contemporary american television and beyond. London: I.B. Tauris.

MCELROY, Wendy. 1995. XXX: A woman's right to pornography. New York: St. Martin's Press.

NEVILLE, Lucy. 2015. « Male gays in the female gaze: Women who watch $\mathrm{m} / \mathrm{m}$ pornography » Porn Studies, 2(2-3): 192-207.

PROULX, Serge \& BÉLANGER, Danielle. 2003 . « La réception des messages » in La communication publique. État des savoir, enjeux et perspectives, GINGRAS, Anne-Marie (éd.). Sainte-Foy : Presses de l'université du Québec, 216-255.

RADWAY, Janice A. 1984. Reading the romance. Women, Patriarchy, and popular literature. Chapell Hill: University of Carolina Press.

ROSALIND, Gill. 2008. « Empowerment/sexism: Figuring female sexual agency in contemporary advertising » Feminism \& Psychology, 18(1): 35-60.

RUBIN, Gayle. 1984. «Thinking sex: Notes for a radical theory of the politics of sexuality » in Pleasure and danger: Exploring female sexuality, VANCE, Carole (éd.). Boston: Routledge, 267-313. RUBIN, Gayle. 2010. Surveiller et jouir : Anthropologie politique du sexe. Paris : EPEL.

SEABROOK, Rita. C. 2017. « Girl Power or Powerless Girl? Television, Sexual Scripts, and Sexual Agency in Sexually Active Young Women » Psychology of Women Quarterly, 41(2): 240-253.

SIMON, Willams \& GAGNON, John 1986. « Sexual scripts: Permanence and change » The Official Publication of the International Academy of Sex Research, 15(2): 97-120.

TIBBALS, Chauntelle Anne. 2014. « Gonzo, trannys, and teens - current trends in US adult content production, distribution, and consumption » Porn Studies, 1(1-2): 127-135.

WILKERSON, Abby, L. 2002. « Disability, Sex Radicalism, and Political Agency » NWSA Journal, 14(3): 33-57.

WILLIAMS, Linda. 1989. Hard core: Power, pleasure, and the "frenzy of the visible". Berkeley: University of California Press.

WILLIAMS, Jean Calterone \& JOVANOVIC, Jasna. 2015. « Third wave feminism and emerging sexuality: Friends with benefits relationships » Sexuality \& Culture, 19(1): 157-171.

\section{ANNEXES}

\section{Filmographie}

ANGEL, Buck, The Buck Stops Here, 2006 [DVD].

ANGEL, Buck, $V$ for Vagina, 2006 [DVD].

BEATTY, Maria, The Black Glove, 1996 [En ligne], consulté le 13 novembre 2015. URL : https://lustcinema.com/movie/the-black-glove

BEATTY, Maria \& DELAIN Rosemary, The Elegant Spanking, 1995 [En ligne], consulté le 4 juillet 2015. URL : https://lustcinema.com/movie/the-elegant-spanking 
BRAMLY, Sophie, Oooooh!, 2013 [En ligne], consulté le 15 août 2018. URL : https:// lustcinema.com/movie/oooooh-

BROWNFIELD, Anne, The Band, 2009 [En ligne], consulté le 10 juillet 2014. URL : https:// lustcinema.com/movie/the-band

COLLECTIF, Dirty Diaries. 12 Shorts of Feminist Porn, 2009 [DVD].

COLLECTIF, X-Femmes, 2010 [DVD].

DAHL, Liandra, Best of Liandra Dahl and Friends vol. 1, 2013 [En ligne], consulté le 10 octobre 2018. URL : https://store.erikalust.com/en/downloads/the-best-of-liandradahl-vol-1

HILL-MEYER, Tobi, Doing it Ourselves, The Trans Women Porn Project, 2010 [DVD].

HOUSTON, Shine Louise, Champion; Love Hurts, 2008 [DVD].

HOUSTON, Shine Louise, Crash Pad, vol. 2, 2007 [DVD].

JOUVET, Émilie, One Night Stand, 2005 [DVD].

LINGHOFT, Lisbeth, Pink Prison, 1999 [DVD].

LUMPKIN, Jincey, Taxi, 2012 [En ligne], consulté le 10 juillet 2015. URL : https://

lustcinema.com/movie/taxi

LUST, Erika, Barcelona Sex Projet, 2008 [DVD].

LUST, Erika, Cabaret Desire, 2011 [DVD].

LUST, Erika, Dude Looks Like a Lady, 2014 [En ligne]. Consulté le 6 septembre 2018. URL : https://xconfessions.com/film/dude-looks-like-a-lady

LUST, Erika, Xconfessions, 2013 [DVD].

LYON BELL, Jennifer, Silver Shoes, 2014 [En ligne], consulté le 17 juillet 2015. URL : https://lustcinema.com/movie/silver-shoes

LYON BELL, Jennifer, Matinée, 2009 [DVD].

LYON BELL, Jennifer \& SCHERRE, Murielle, Skin Like Sun. Des jours plus belles que la nuit, 2009 [DVD].

NILSSON, Jessica, All About Anna, 2005 [DVD].

OVIDIE \& TYLER, Jack, Sex Stories- Histoires de sexe(s), 2010 [DVD].

OVIDIE, Liberté sexuelle, 2012 [En ligne], consulté le 5 septembre 2015. URL : https:// lustcinema.com/movie/liberte-sexuelle

ROYALLE, Candida, Stud Hunters, 2002 [En ligne], consulté le 5 novembre 2015. URL : https://lustcinema.com/movie/stud-hunters

ROYALLE, Candida, Eyes of Desire 1, 1998 [En ligne], consulté le 5 novembre 2015. URL : https://lustcinema.com/movie/ eyes-of-desire-1

ROYALLE, Candida, Eyes of Desire 2, 1999 [En ligne], consulté le 10 août 2015. URL :

https://lustcinema.com/movie/eyes-of-desire-2 
TAORMINO, Tristan, Expert Guide to Anal Pleasure for Men, 2009 [En ligne], consulté le 11 novembre 2015. URL : https://lustcinema.com/movie/expert-guide-to-anal-pleasurefor-men

TROUBLE, Courtney, Billy Castro Does the Mission, 2010 [DVD].

TROUBLE, Courtney, Trans Grrrls, 2013 [DVD].

TROUBLE, Courtney \& BIKE SMUT, Come Find Me, suivi de Pedals for Pleasure, 2013 [DVD].

TROUBLE, Courtney, Speakeasy, 2009 [DVD].

TROUBLE, Courtney, Bordello, 2009 [DVD].

TROUBLE, Courtney, Nostalgia, 2009 [DVD].

YOUNG, Madison, Fluid: Men Redefining Sexuality, 2009 [DVD].

YOUNG, Madison, Fluid: Women Redefining Sexuality, 2009 [DVD].

YOUNG, Madison, 50 Shades of Dylan Ryan, 2012 [DVD].

\section{NOTES}

1. Par «script hétérosexuel ", nous faisons référence à la théorie des scripts sexuels développée par Simon et Gagnon en 1973 qui conçoit la sexualité comme déterminée et apprise à travers des scripts plus ou moins élaborés codifiés comme sexuels.

2. Par ailleurs, il importe de nuancer le fait que bien que les femmes apprécient la pornographie mainstream (McElroy, 1995) comme la pornographie gaie (Neville, 2015), la pornographie ciblée dans ce projet explicite s'adresse plus directement à un public de femmes ou à un public queer. De même, nous sommes conscientes que le sujet politique «femme » que soutient ce projet est fondamentalement fictionnel: d'un côté, certaines pornographies se revendiquent d'une pornographie par et pour des femmes cis ou trans, et d'autre part, certains projets plus politiques et queer remettent en question directement le sujet femme comme performance à subvertir.

3. Traduction québécoise du hashtag \#metoo.

4. Selon Foucault (1976), la scientia sexualis est un dispositif de l'Occident qui vise à produire un savoir-pouvoir sur la " vérité du sexe » à partir des procédures de l'aveu (p. 78). Il s'oppose à l'ars erotica (présent en Chine, au Japon, en Inde...) qui vise à extraire la vérité de l'expérience du plaisir.

5. Clarissa Smith et Feona Attwood (2013) ont identifié un retour du féminisme antipornographie à partir des années 2000. Ce retour initié par Gail Dines notamment s'attaque non seulement à la pornographie, mais également à la pornographisation de la culture.

6. On n'a qu'à penser aux épithètes injurieuses et aux niches pornographiques shemale ou tranny issues de la pornographie mainstream qui contribuent à fétichiser et déshumaniser les femmes trans (Tobi Hill-Meyer 2013).

7. Suicidegirls.com est un site de pornographie alternative ayant vu le jour en 2001. Créée par Missy Suicide (Selena Mooney) et Sean Suhl, la plateforme diffuse des photos érotiques de femmes adeptes de modifications corporelles inspirées des cultures et des esthétiques punk, emo, gothique ou pinup. À l'origine, il s'agissait d'un projet voulant valoriser la beauté non conformiste des femmes qui arborent tatouages, perçages et cheveux colorés, puisqu'à l'époque s'afficher ainsi pour une femme consistait en une forme de "suicide social » en raison de la stigmatisation associée à ces pratiques corporelles. Près de vingt ans plus tard, Suicide Girls est aujourd'hui critiqué pour sa «mainstreamisation» et le recours aux retouches photos. Pour sa 
part, furrygirl.com est l'initiative de Furry Girl qui est à la fois modèle et gestionnaire du site web. Actif entre 2003 et 2015 , le site diffuse du contenu visuel photo (près de 10000 photos érotiques) et vidéo réalisé et autoproduit par Furry Girl. Son esthétique s'appuie sur la valorisation de son corps au naturel, c'est-à-dire en l'absence de maquillage et avec sa pilosité naturelle (jambes, aisselles et toison pubienne à leur état original), le tout dans une facture pornographique amatrice.

8. La moitié des films ont été achetés en format DVD alors que l'autre moitié a été obtenue via l'abonnement au cinéma pornographique féministe en ligne Lust cinema qui offre à ses membres un accès à de nombreux longs et courts métrages pornographiques.

9. 50 Shades of Dylan Ryan est un porno BDSM lesbien inspiré de la trilogie Fifty Shades of Grey. Dans le film, Mme Grey est une riche femme d'affaires de l'industrie du livre. Elle vient de publier un livre intitulé 50 Shades of Submission (ici le schème narratif est identique à celui du livre original). Dylan critique ouvertement le livre d'être antiféministe, car il perpétue des fantasmes misogynes, en plus du risque de nuire aux communautés BDSM en raison de sa représentation peu réaliste. Elle fait le pari de s'immerger dans la sous-culture BDSM et d'être l'esclave sexuelle de Mme Grey durant une semaine, avec la tâche de documenter l'authenticité de son expérience auprès du public. Si le public préfère les chroniques de Dylan à l'histoire de 50 Shades of Submission, alors Mme Grey se voit dans l'obligation de publier un livre sur les sous-cultures sexuelles aux États-Unis.

10. Nous faisons ici référence à un type de pornographie faite par et pour lesbiennes qui se distingue clairement du « girl on girl » de la pornographie commerciale hétérosexuelle.

11. Un des collectifs correspond aux deux sous-groupes.

\section{RÉSUMÉS}

Dans les débuts de la commercialisation de pornographie hardcore, la pornographie hétérosexuelle met en scène une sexualité généralement non simulée où le plaisir des femmes est secondaire par rapport à celui de l'homme. En réaction à cette production, s'est développée une pornographie féministe qui place au centre de ses réalisations la sexualité des femmes cis ou des personnes trans, qu'elles soient hétérosexuelles, lesbiennes, bisexuelles, fluides, pansexuelles ou queer. Nous avons analysé un corpus de 38 films pornographiques féministes afin de dégager les scripts sexuels mis en scène. Cet article cible la question de l'agentivité sexuelle des femmes, opérationnalisée en trois marqueurs : l'expression du consentement, l'expression des préférences sexuelles et la remise en question du script hétérosexuel traditionnel. L'analyse des films composant notre corpus nous permet de confirmer la présence accrue de représentations de femmes agentives sexuellement.

In the early days of the commercialization of hardcore pornography, heterosexual pornography portrays a generally unfaked sexuality in which women's pleasure is subordinated. In reaction to this production, feminists have created a pornography that foregrounds the sexuality of cis women or trans people (lesbians, bisexuals, fluids, pansexuals, heterosexuals or queer). We have analyzed a corpus of 38 feminist pornographic films in order to identify sexual scripts. The analysis focuses on the question of women's sexual agency, operationalized in three markers: the expression of consent, sexual preferences and the transformation of the traditional heterosexual 
script. The analysis of the selected films allowed us to confirm the presence of representations of women's sexual agency.

\section{INDEX}

Thèmes : Recherches

Mots-clés : pornographies féministes, agentivité sexuelle, théorie des scripts sexuels, représentation de la sexualité

Keywords : feminist pornographies, sexual agency, sexual script theory, sexual representation

\section{AUTEURS}

\section{JULIE LAVIGNE}

Julie Lavigne est historienne de l'art et professeure au Département de sexologie de l'Université du Québec à Montréal (UQAM). Elle participe à titre de membre régulière au Réseau québécois en études féministes (RéQEF). Elle a publié l'ouvrage La traversée de la pornographie. Politique et érotisme dans l'art féministe aux éditions du Remue-ménage.

\section{MYRIAM LE BLANC ELIE}

Myriam Le Blanc Élie a travaillé durant plus de trois ans sur le projet Pornographie critique, féministe, queer et post-pornographie : contours d'une pratique émergente mené par Julie Lavigne. Elle exerce actuellement auprès d'adolescent-e-s et mène un projet de prévention des cyberviolences sexuelles.

\section{SABRINA MAIORANO}

Sabrina Maiorano est candidate au doctorat en sexologie à l'Université du Québec à Montréal. Son projet de thèse porte sur l'analyse des discours sexopolitiques des pornographies lesbiennes BDSM. Elle travaille principalement sur l'agentivité sexuelle et porte un intérêt grandissant à la place accordée aux affects dans la théorisation féministe de la pornographie et sa réception. 